OAK RIDGE

NATIONAL LABORATORY

MANAGED BY UT-BATTELLE

FOR THE DEPARTMENT OF ENERGY

\title{
SIMULATED NMIS IMAGING DATA FOR AN UNKNOWN OBJECT
}

\section{April 2012}

Prepared by

Mark E. Walker

John T. Mihalczo

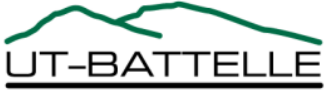




\section{DOCUMENT AVAILABILITY}

Reports produced after January 1, 1996, are generally available free via the U.S. Department of Energy (DOE) Information Bridge.

Web site http://www.osti.gov/bridge

Reports produced before January 1, 1996, may be purchased by members of the public from the following source.

National Technical Information Service

5285 Port Royal Road

Springfield, VA 22161

Telephone 703-605-6000 (1-800-553-6847)

TDD 703-487-4639

Fax 703-605-6900

E-mail info@ntis.gov

Web site http://www.ntis.gov/support/ordernowabout.htm

Reports are available to DOE employees, DOE contractors, Energy Technology Data Exchange (ETDE) representatives, and International Nuclear Information System (INIS) representatives from the following source.

Office of Scientific and Technical Information

P.O. Box 62

Oak Ridge, TN 37831

Telephone 865-576-8401

Fax 865-576-5728

E-mail reports@osti.gov

Web site http://www.osti.gov/contact.html

This report was prepared as an account of work sponsored by an agency of the United States Government. Neither the United States Government nor any agency thereof, nor any of their employees, makes any warranty, express or implied, or assumes any legal liability or responsibility for the accuracy, completeness, or usefulness of any information, apparatus, product, or process disclosed, or represents that its use would not infringe privately owned rights. Reference herein to any specific commercial product, process, or service by trade name, trademark, manufacturer, or otherwise, does not necessarily constitute or imply its endorsement, recommendation, or favoring by the United States Government or any agency thereof. The views and opinions of authors expressed herein do not necessarily state or reflect those of the United States Government or any agency thereof. 
Global Nuclear Security Technology Division

\title{
SIMULATED NMIS IMAGING DATA FOR AN UNKNOWN OBJECT
}

\author{
Mark E. Walker \\ John T. Mihalczo
}

Date Published: April 2012

\author{
Prepared by \\ OAK RIDGE NATIONAL LABORATORY \\ P.O. Box 2008 \\ Oak Ridge, Tennessee 37831-6285 \\ managed by \\ UT-BATTELLE, LLC \\ for the \\ US DEPARTMENT OF ENERGY \\ under contract DE-AC05-00OR22725
}





\section{CONTENTS}

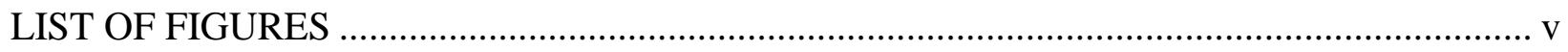

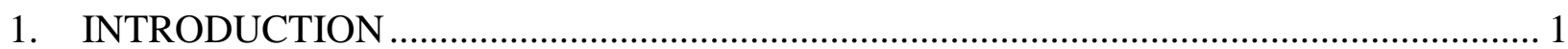

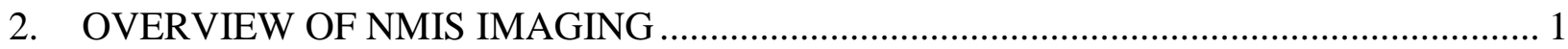

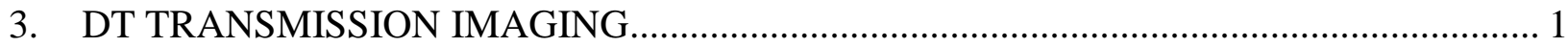

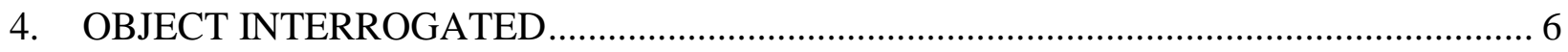

APPENDIX A. IMAGING DATA FILES ………………............................................... A-1 



\section{LIST OF FIGURES}

Fig. $\quad$ Page

1 Schematic of particle tagging in the DT generator (not to scale)................................... 2

2 Schematic of a transmission measurement with NMIS................................................ 2

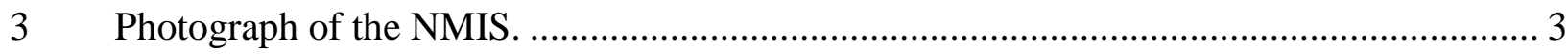

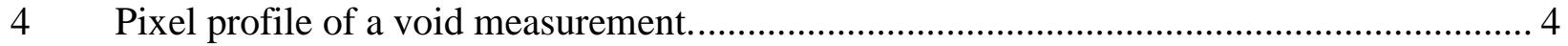

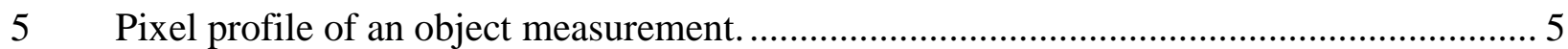

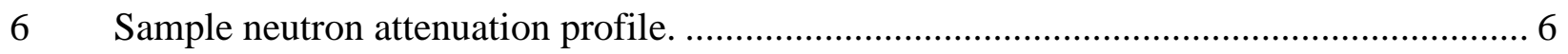





\title{
SIMULATED NMIS IMAGING DATA FOR AN UNKNOWN OBJECT
}

\author{
Mark E. Walker and John T. Mihalczo
}

\section{INTRODUCTION}

This report presents simulated Nuclear Materials Identification System (NMIS) imaging data for an unclassified object, whose characteristics are initially unknown to the reader. This data will be used to test various analysis capabilities and was created with a simple deterministic ray-tracing algorithm.

\section{OVERVIEW OF NMIS IMAGING}

NMIS is a time-dependent coincidence counting system that is used to characterize both fissile and non-fissile materials undergoing nondestructive assay. NMIS characterizes materials by interrogating them with neutrons, either from an associated-particle deuterium-tritium (DT) neutron generator, which produces a time and directionally tagged monoenergetic beam of 14.1 MeV neutrons, or a time-tagged spontaneous fission source in an ionization chamber.

\section{DT TRANSMISSION IMAGING}

The DT transmission imaging technique involves measuring the attenuation of neutrons from a DT generator through an object. Neutrons are time and directionally tagged at the DT generator with an embedded alpha particle detector, and neutrons that are transmitted through the object are detected in a horizontal plastic scintillator fan array, which is $115 \mathrm{~cm}$ away from the neutron generator. For this analysis, a simple ray-tracing algorithm provides time coincidence data between the scintillator array and the neutron generator alpha pixels, and produces a horizontal tomograph that maps neutron attenuation through the object.

The DT generator produces neutrons by accelerating deuterium and tritium molecules, under a potential of $90 \mathrm{kV}$, into a metal hydride target, which also contains deuterium and tritium. The resulting fusion reactions between deuterium and tritium produce alpha particles and $14.1 \mathrm{MeV}$ neutrons, which are emitted in essentially opposite directions (the momentum from the accelerated molecules shifts the angular distribution by about 5 degrees). Embedded in the generator is a scintillator adjacent to a fiber-optic face plate, whose outside face is adjacent to a pixelated light guide. The light guide is connected to a pixelated photomultiplier tube (PMT). This setup allows for neutrons to be time and directionally tagged through the detection of their associated alpha particles (Fig. 1) across 16 separate pixels. 


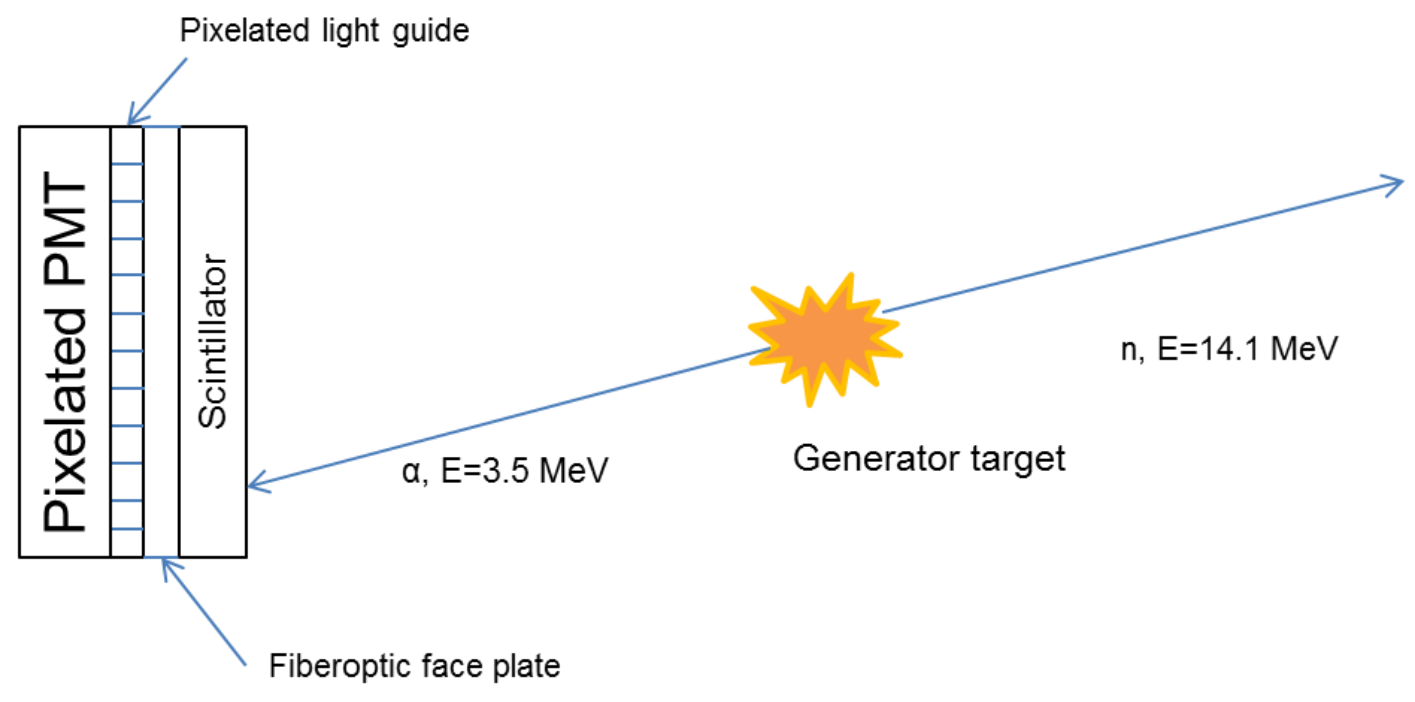

Fig. 1. Schematic of particle tagging in the DT generator (not to scale).

Tagged neutrons subsequently interact with the object that is being measured. Neutrons that are transmitted through the object, as well as products of neutron interactions inside the object, become incident on a horizontal fan array of 32 sequentially numbered plastic scintillators. A schematic of possible interactions is shown in Fig. 2, and a photograph of the NMIS system is shown in Fig. 3. Directly transmitted (unattenuated) neutrons arrive at the scintillator array $22.5 \pm 1 \mathrm{~ns}$ after the neutron is emitted by the target. Neutrons that elastically scatter in the object arrive later, as well as neutrons produced from induced fission inside the object.

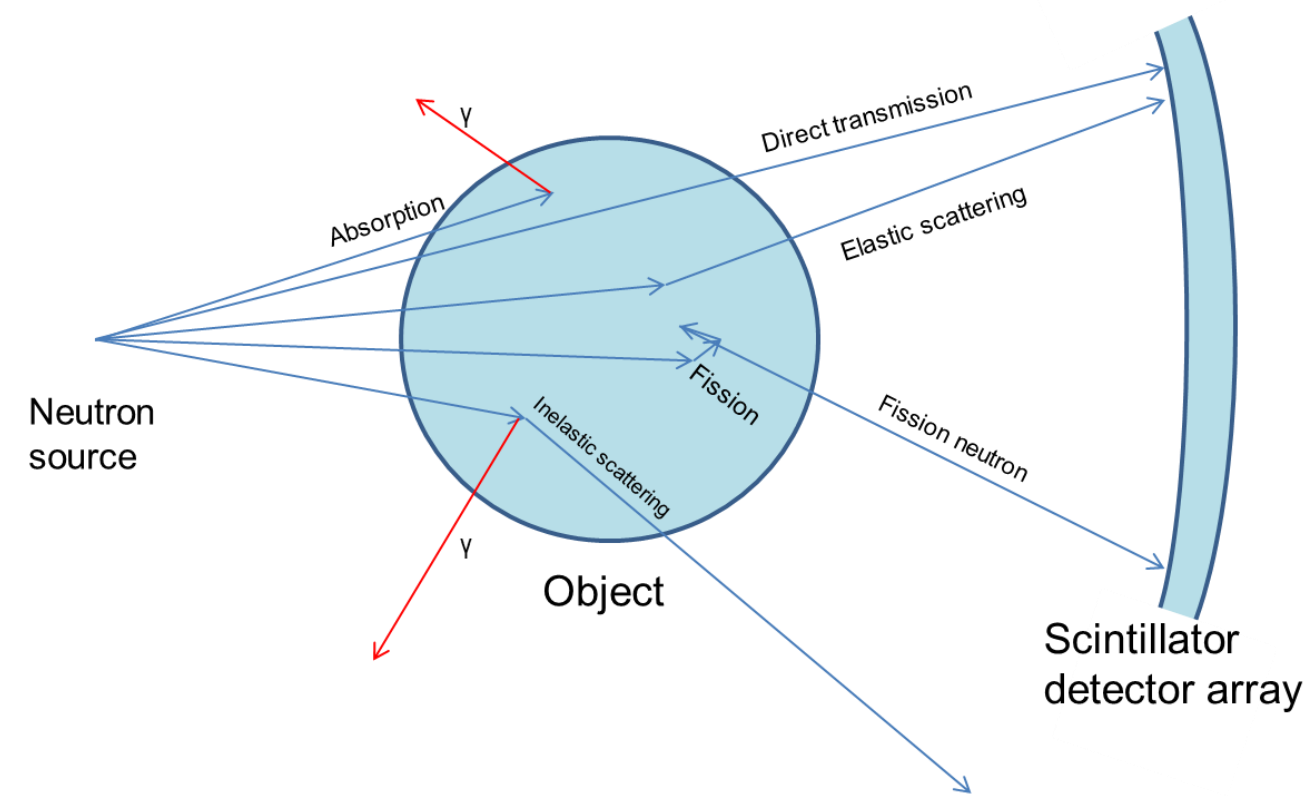

Fig. 2. Schematic of a transmission measurement with NMIS. 


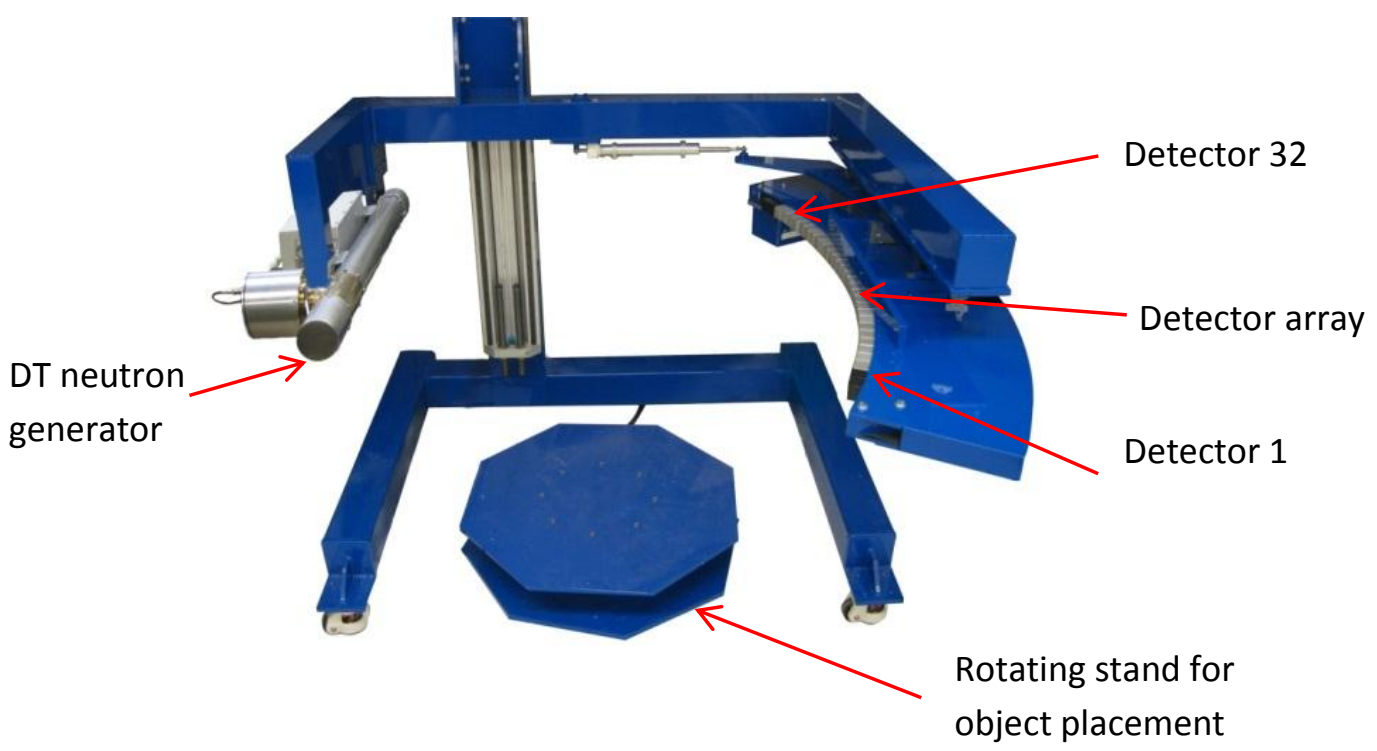

Fig. 3. Photograph of the NMIS.

The signal from each pixel in the DT generator is connected to a constant fraction discriminator (CFD), whose threshold is individually set so that counts rates in the 16 pixels are approximately equal. The widths of the output logic pulses for each CFD are set to different levels, so that when the signals are merged, pulses in each individual pixel are distinguishable. The signals from the odd-numbered pixels are merged in a fan-in/fan-out module, and the signals from the evennumbered pixels are merged in another fan-in/fan-out module. The outputs of these modules, respectively, compose the inputs to channels 1 and 6 of a 10-channel, 1 GHz NMIS data acquisition and processing board.

Signals from the plastic scintillator detectors are processed in a similar manner. Each detector signal is processed through a CFD, whose threshold is usually set to $\sim 1 / 3$ of the full energy peak when a Cs-137 source is placed on the detector. The pulse widths in the CFD outputs are set so that pulses in each detector are distinguishable when signals are merged in fan-in/fan-out modules. Signals from the plastic scintillator array compose the inputs to channels 2, 3, 4, 7, 8, and 9 of the NMIS data acquisition and processing board.

Since channels 1-5 and 6-10 are sent to different NMIS processor boards, a timing synchronization signal is sent to both boards. This is required because each processor board has a separate clock. In this case a preamplifier with no input is sent to a CFD having a very low threshold so that preamplifier noise causes the CFD to emit logic noise pulses randomly. The CFD output is split, with one signal transmitted to channel 5 of the NMIS board and the other signal sent to channel 10. The NMIS processor is programmed to synchronize the times of channels $1-5$ with channels $6-10$ by adjusting the time stamp for channels 6-10.

Analysis of data from NMIS is accomplished with the Data Acquisition User Interface (DAUI) system. For every NMIS measurement, a detector system data (*.DSD) file must be used as an 
input for DAUI. This file contains the set pulse widths for every detector in the system, as well as the delay time associated with cable lengths between the detectors. Other DAUI inputs include spatial data, such as the distance from the neutron generator target to the center of the object. The NMIS detector arms can be raised and lowered automatically, and the object can be rotated on a turntable. The plastic scintillator array can also be moved side-to-side between measurement samples, which improves spatial resolution in the image. The motors that control these three functions are programmed through the DAUI interface.

After a measurement is completed, DAUI produces a *.NMD file containing coincidence data between all of the detectors. This data can be analyzed to produce a "pixel profile" in an Excel *.csv format. A set of 11 alpha pixel profiles accompany this report and are found in Appendix A. These profiles were produced in simulated measurements. An example of a pixel profile for a "void" measurement with no object present is shown in Fig. 4, and a pixel profile measurement for an object measurement is shown in Fig. 5.

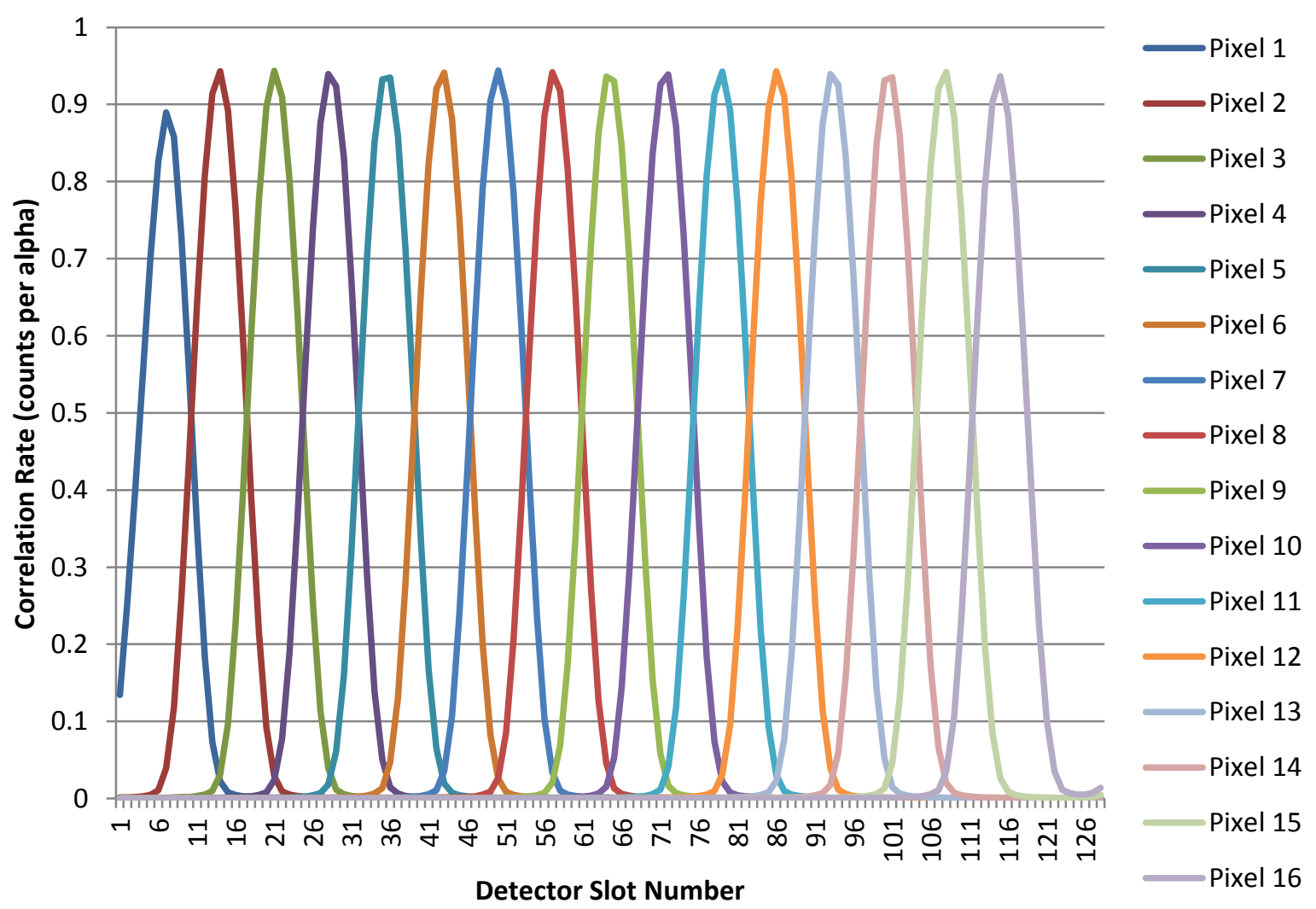

Fig. 3. Pixel profile of a void measurement. 


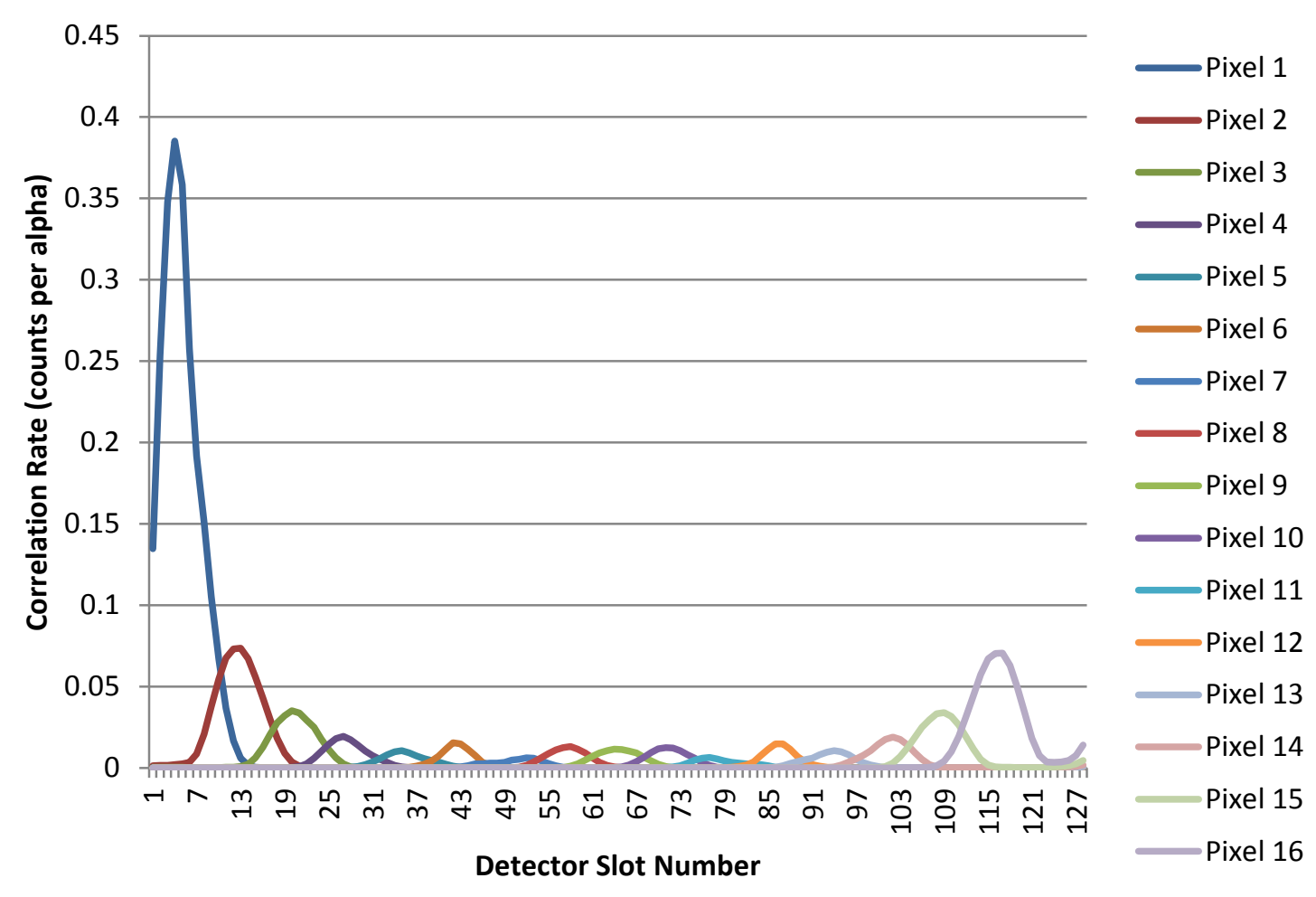

Fig. 4. Pixel profile of an object measurement.

Each number in these *.csv spreadsheets corresponds to the coincidence rate between an alpha pixel detector and a plastic scintillator detector. This rate is given in counts per alpha particle detected. Rows in the *.csv files correspond sequentially to alpha pixels, and the columns correspond to slots in the imaging detector array. Each detector slot represents $1 / 4 \mathrm{in}$. of displacement of the front face of $1 \times 1 \times 4$-in.-thick imaging detector along an arc. The plastic scintillator array was placed in four positions through the course of the simulation, for a total of 128 detector positions.

As an example, a number given in cell B1 is the number of neutrons that are detected in detector slot 2 over the course of the measurement, divided by the number of alpha particles detected in pixel 1 over the same time frame. The intrinsic efficiency of the imaging detectors, for these simulations, is assumed to be perfect to provide a best-case scenario.

Neutron attenuation can be calculated for each detector slot with the equation

$$
\text { attenuation }=-\ln \left(\frac{I}{I_{0}}\right) \text {, }
$$

where $I$ is the total correlation rate of a detector slot with an object present, and $I_{0}$ is the total correlation rate of the same detector slot with no object present. Correlation rates are determined by integrating the timing peak centered on the expected time of arrival for unattenuated 14.1 MeV neutrons. A total correlation rate for a detector slot can be calculated by summing all 
of its pixel correlation rates (summing each column will produce a total correlation rate for each detector slot). A sample neutron attenuation profile for an object is shown in Fig. 6.

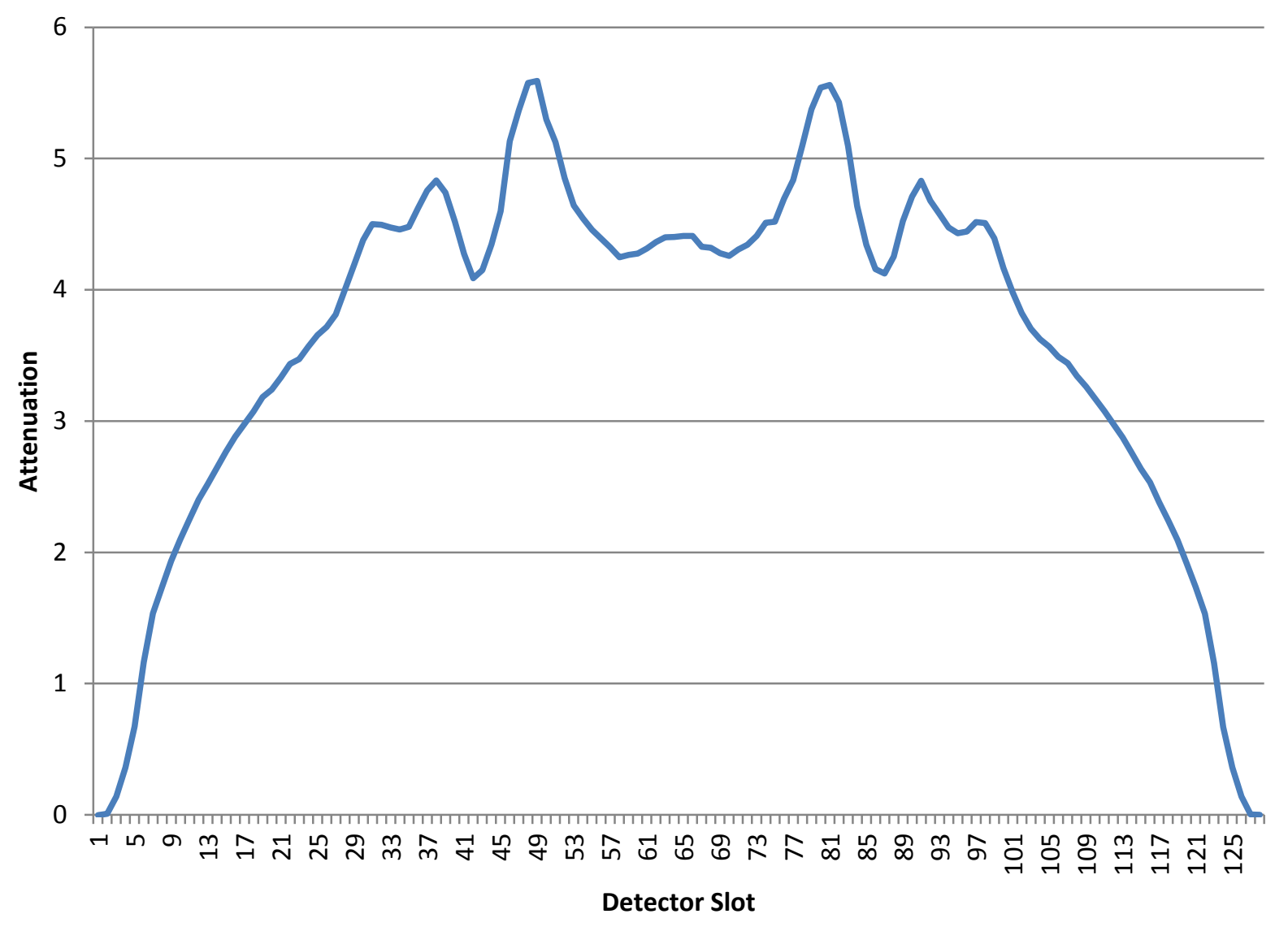

Fig. 5. Sample neutron attenuation profile.

The file "void_1_NMIS.csv" contains data for a measurement with no object present. This "void" file serves to provide an unattenuated pixel profile to provide $I_{0}$ values for calculating neutron attenuation. "obj1_1_NMIS.csv" contains data for the unperturbed object. The other files with names "obj*_1_NMIS.csv" contain data for perturbations of the imaging.

\section{OBJECT INTERROGATED}

The object interrogated is an unclassified cylindrically symmetric object, whose center is $55 \mathrm{~cm}$ from the neutron generator's target, along the centerline between the neutron target and the middle of the plastic scintillator array. The neutron target is assumed to be a point. The image is at the midplane and a significant distance from the top and bottom of the object. 



\section{APPENDIX A. IMAGING DATA FILES}

This appendix contains the simulated data files for the case with no object between the source and the detectors, with the object in place with the axis of the object $55 \mathrm{~cm}$ from the source, and unspecified perturbations of the system. The axis of the cylindrically symmetric system is perpendicular to the horizontal fan array of imaging detectors and the row of alpha pixels. The 128 detector slots correspond to four locations of the imaging array, each $1 / 4 \mathrm{in}$. apart. Because of the deterministic nature of the algorithm used to produce the data, statistics may be assumed to be perfect. Links to each file are found below.

Pixel profile of void measurement:

Pixel profile of unperturbed object:

Pixel profiles of perturbed objects:
void_1_NMIS.csv

obj1_1_NMIS.csv

obj2_1_NMIS.csv

obj3_1_NMIS.csv

obj4_1_NMIS.csv

obj5_1_NMIS.csv

obj6_1_NMIS.csv

obj7_1_NMIS.csv

obj8_1_NMIS.csv

obj9_1_NMIS.csv

obj10_1_NMIS.csv

Neutron attenuation profile of unperturbed object: $\quad$ Object 1 Neutron Attenuation.xlsx 